\title{
Use of the melting curve assay as a means for high- throughput quantification of Illumina sequencing libraries
}

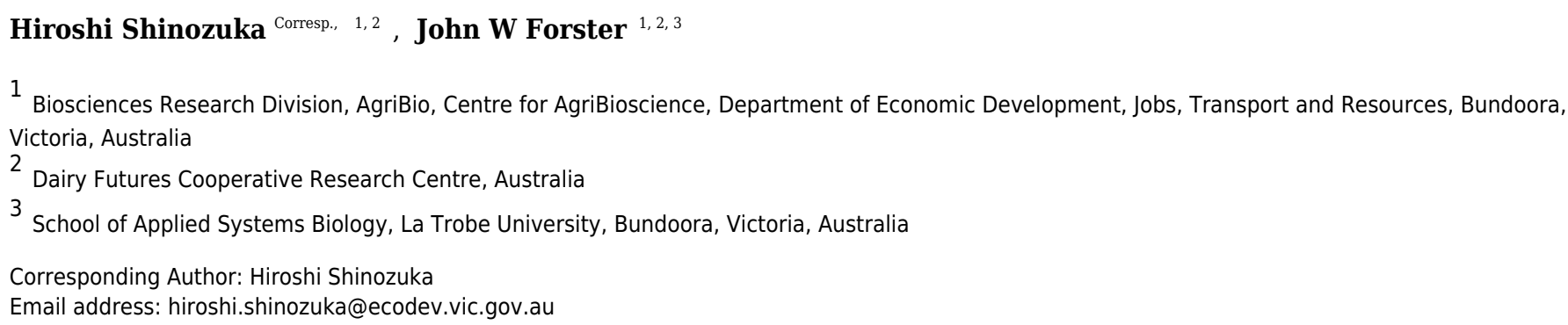

Background. Multiplexed sequencing is commonly performed on massively parallel shortread sequencing platforms such as Illumina, and the efficiency of library normalisation can affect the quality of the output dataset. Although several library normalisation approaches have been established, none is ideal for highly multiplexed sequencing due to issues of cost and/or processing time. Methods. An inexpensive and high-throughput library quantification method has been developed, based on an adaptation of the melting curve assay. Sequencing libraries were subjected to the assay using the Bio-Rad Laboratories CFX Connect ${ }^{T M}$ Real-Time PCR Detection System. The library quantity was calculated through summation of reduction of relative fluorescence units between 86 and $95^{\circ} \mathrm{C}$. Results. PCR-enriched sequencing libraries are suitable for this quantification without prepurification of DNA. Short DNA molecules, which ideally should be eliminated from the library for subsequent processing, were differentiated from the target DNA in a mixture on the basis of differences in melting temperature. Quantification results for long sequences targeted using the melting curve assay were correlated with those from existing methods $\left(R^{2}>0.77\right)$, and that observed from MiSeq sequencing $\left(R^{2}=0.82\right)$. Discussion. The results of multiplexed sequencing suggested that the normalisation performance of the described method is equivalent to that of another recently reported high-throughput bead-based method, BeNUS. However, costs for the melting curve assay are considerably lower and processing times shorter than those of other existing methods, suggesting greater suitability for highly multiplexed sequencing applications. 
1 Use of a melting curve assay as a means for high-throughput

2 quantification of Illumina sequencing libraries

4 Hiroshi Shinozuka ${ }^{1,2,{ }^{*}}$ and John W. Forster ${ }^{1,2,3}$

6 'Department of Economic Development, Jobs, Transport and Resources, 7 Biosciences Research Division, AgriBio, Centre for AgriBioscience, 5 Ring Road,

8 La Trobe University, Bundoora, Victoria 3083, Australia

9 2Dairy Futures Cooperative Research Centre, Australia

$10{ }^{3}$ School of Applied Systems Biology, La Trobe University, Bundoora, Victoria

11 3086, Australia

$12 \quad$ *Corresponding author: e-mail - hiroshi.shinozuka@ecodev.vic.gov.au

13 Keywords: second-generation sequencing technology; multiplexed sequencing;

14 library preparation; library normalisation 


\section{Abstract}

Background. Multiplexed sequencing is commonly performed on massively parallel short-read sequencing platforms such as Illumina, and the efficiency of library normalisation can affect the quality of the output dataset. Although several library normalisation approaches have been established, none is ideal for highly multiplexed sequencing due to issues of cost and/or processing time.

Methods. An inexpensive and high-throughput library quantification method has been developed, based on an adaptation of the melting curve assay. Sequencing libraries were subjected to the assay using the Bio-Rad Laboratories CFX Connect ${ }^{\mathrm{TM}}$ Real-Time PCR Detection System. The library quantity was calculated through summation of reduction of relative fluorescence units between 86 and $95^{\circ} \mathrm{C}$.

Results. PCR-enriched sequencing libraries were suitable for this quantification without pre-purification of DNA. Short DNA molecules, which ideally should be eliminated from the library for subsequent processing, were differentiated from the target DNA in a mixture on the basis of differences in melting temperature. Quantification results for long sequences targeted using the melting curve assay were correlated with those from existing methods $\left(R^{2}>0.77\right)$, and that observed from MiSeq sequencing $\left(R^{2}=0.82\right)$.

Discussion. The results of multiplexed sequencing suggested that the normalisation performance of the described method is equivalent to that of another recently reported high-throughput bead-based method, BeNUS. However, costs for the melting curve assay are considerably lower and processing times shorter than those of other existing methods, suggesting greater suitability for highly multiplexed sequencing applications. 
38

39

40

41

\section{Introduction}

Substantial reductions in both the cost and processing time of DNA sequencing have been achieved in the last decade, due to improvements of massively parallel short-read sequencing technologies, and this trend is expected to continue for the next several years or more (http://ark-invest.com/genomic-revolution/declining-costs-of-genomesequencing). As a consequence, sequencing library preparation procedures, rather than the DNA sequencing process itself, provide the major bottleneck for large-scale experimentation (Rohland \& Reich, 2012). As a cost-effective approach, single sequencing libraries are parallel-processed from individual samples, and assigned a unique oligonucleotide index. The libraries are then pooled for multiplexed processing in a single run (or lane) of a high-throughput sequencing platform. During this pooling step, equalisation of DNA quantity from each library is essential for an optimised sequencing output (Hosomichi et al., 2014).

Although several library normalisation methods have been established, all of them incur a relatively high cost and/or require long processing times for a large number of samples (Table 1, Supplementary Table 1) (Buehler et al., 2010; Campbell, Harmon \& Narum, 2015; Harris et al., 2010; Hosomichi et al., 2014; Katsuoka et al., 2014; Kong et al. 2014; Rohland \& Reich, 2012). Single libraries are commonly quantified with a well-established real-time PCR-based method (Buehler et al., 2010; Rohland \& Reich, 2012) which, however, requires a relatively long processing time. The qMiSeq method was recently proposed as a highly accurate library titration method for multiplexed sequencing libraries (Katsuoka et al., 2014). In this method, the pooled library is first sequenced on the MiSeq instrument (Illumina, CA, USA) in order to estimate the quantity of single libraries, which are then re-pooled for sequencing on the HiSeq 
62 platform (Illumina). Although this method is highly efficient when applied to an extensive

63 sequencing experiment for an organism with a large genome, the per-sample cost approaches US\$10 if the sample number is 93 or equivalent.

Melting curve (MC) assays identify the melting temperature (MT) point at which double-stranded DNA (dsDNA) dissociates into single-stranded DNA (ssDNA), through detection of reductions in dsDNA-specific fluorescence signals during the heating process (Ririe, Rasmussen \& Wittwer, 1997). This assay can be performed on most real-time PCR instruments, and up to 96-1536 samples can be simultaneously processed, depending on the instrument format (Lennon et al. 2010). MT largely depends on the length of dsDNA, such that shorter dsDNA molecules, in general, dissociate at lower temperatures than longer ones (Ririe, Rasmussen \& Wittwer, 1997). As a consequence, the MC assay is commonly used for quality control purposes in realtime PCR experiments that show presence/absence of undesirable short amplicons, such as PCR primer dimers.

An application of the MC assay for quantification of sequencing libraries is reported in this study. Libraries for multiplexed sequencing are generally enriched through PCR, and short DNA molecules, such as PCR primers, primer dimers, and amplicons derived from self-ligated sequencing adapters must be excluded from quantification. Although SPRI (solid phase reversible immobilisation)-based DNA sizeselection is commonly used for this purpose (Harris et al., 2010), this approach requires a relatively large amount of consumables (micropipette tips and microtiter plates) and a long processing time. This procedure is not required for MC assay-based quantification, which permits exclusion of such short DNA molecules from library quantification, based on differences of MT. In addition, as real-time PCR instruments are commonly present 
86 in molecular biology laboratories, this normalisation approach can be readily applied in 87 labs and hence may not require the acquisition of a new instrument. 
89

90

91

92

93

94

95

96

\section{Materials \& Methods}

0

\section{Preparation of short DNA and KAPA fragments}

In order to generate short DNA amplicons (<300 bp), which are normally eliminated from sequencing libraries, an in-house library procedure was performed in the absence of DNA template. An in-house-designed double-stranded Y-shaped adaptor (Supplementary Figure S1) with a single thymine base extension (50 pmol) was treated with the Klenow fragment of Escherichia coli DNA polymerase I (conferring $3^{\prime} \rightarrow 5^{\prime}$ exonuclease activity) (New England Biolabs; NEB, MA, USA) in the presence of dATP, followed by self-ligation using T4 DNA ligase (NEB). The ligated products were purified with Lambda Exonuclease and Exonuclease I (NEB), and then subjected to PCR amplification for 32 cycles with the Phusion DNA polymerase kit (Thermo Fisher scientific, MA, USA) and in-house library preparation primers containing sequencing indexes. PCR amplification was also performed using the DNA standard 3, primer mix and qPCR master mix of the KAPA Library Quantification Kit Illumina Platforms (Kapa Biosystems, MA, USA), to generate amplicons of $452 \mathrm{bp}$ in length. Sample mixtures (total volume: $20 \mu \mathrm{l}$ ), containing the short DNA or KAPA fragments, 1x PCR buffer, and SYBR Green I (Thermo Fisher Scientific), were prepared for the MC assay.

\section{Preparation of sequencing libraries}

Sequencing libraries for the MiSeq platform were prepared following a previously described high-throughput method based on use of the methylation-dependent restriction endonuclease MspJI (NEB) (Shinozuka et al. 2015). Genomic DNA of strains of the most prevalent perennial ryegrass-associated fungal endophyte (Epichloë 
113 festucae var. Iolii), of which genome size is around $30 \mathrm{Mb}$, was directly amplified from

114 mycelium using the REPLI-g Mini Kit (QIAGEN, Hilden, Germany). In the amplification

115 solution of the kit, $16.7 \mu \mathrm{M} 5$-methylcytosine (TriLink biotechnologies, CA, USA) was

116 included. The amplicons were digested with MspJI in order to promote semi-random

117 DNA fragmentation. The DNA fragments were treated with the Klenow fragment,

118 followed by ligation of the in-house-designed adaptor using T4 DNA ligase. The DNA

119 was then purified and size-selected using AMPure XP beads (Beckman Coulter, CA,

120 USA), a portion of which was used for PCR enrichment with the in-house-designed

121 primers using the Phusion polymerase kit. During this process, a unique sequencing

122 string was attached to each sample. Using SYBR Green I as a fluorescence dye, 123 amplification was monitored on the CFX Connect ${ }^{\mathrm{TM}}$ Real-Time PCR Detection System

124 (Bio-Rad Laboratories, CA, USA).

125

126

127

128

129

130

131

132

133

134

135

136

\section{MC assay-based dsDNA quantification}

For the MC assay-based quantification procedure, $10 \mu \mathrm{l}$ of DNA staining mixture, containing $0.1 \mu \mathrm{l}$ 100x SYBR Green I, $2 \mu \mathrm{l}$ 5x PCR buffer, $1.5 \mu \mathrm{l}$ 500mM EDTA and 6.4 $\mu \mathrm{l}$ PCR-grade water, was prepared and added to 20 or $25 \mu \mathrm{l} \mathrm{PCR}$ products. The MC assay was performed on the real-time PCR instrument. Initial sample heating was performed at $75^{\circ} \mathrm{C}$ for $30 \mathrm{sec}$, and then samples were incubated at each degree point for 5 or $10 \mathrm{sec}$, followed by regular temperature increments of a single degree. Fluorescence measurement was performed after incubation at each degree point. The library concentration was calculated through summation of reduction of relative fluorescence units (dRFU) between 86 and $95^{\circ} \mathrm{C}$. 


\section{Automated gel electrophoresis-based DNA quantification}

138 Library quantification was performed with the 2200 TapeStation Instrument and the

139 D1000 kits (Agilent Technologies, CA, USA). In order to reduce technical error, $2 \mu$ l of 140 PCR product was added to $6 \mu$ L1000 Reagent, instead of $1 \mu$ LNA sample and $3 \mu l$

141 D1000 Reagent as described in the manufacturer's instruction. Quantity of DNA 142 between $300-700$ bp in length was manually determined and expressed as library 143 quantity.

\section{NanoDrop-based DNA quantification}

146

147

148

149

150

151

152

153

154

155

156

157

158

159

PCR products were purified with 0.8 times volume of AMPure XP beads in order to remove DNA fragments shorter than $300 \mathrm{bp}$ in length, and DNA was eluted in 10mM Tris-HCl buffer. The concentration of purified DNA was measured on the NanoDrop 1000 instrument (Thermo Fisher Scientific).

\section{Massively-parallel short-read sequencing}

Sequencing libraries were pooled based on the basis of quantification result. The pooled library was purified using 0.8 times volume of AMPure XP beads, and subsequently characterised with the TapeStation and Qubit instruments (Thermo Fisher Scientific). The pooled library was loaded and sequenced on the Illumina MiSeq platform, following the manufacturer's instruction. Output data were analysed using the PRINSEQ-Lite software (Schmieder \& Edwards, 2011; http://prinseq.sourceforge.net/). The percentage of paired-end read numbers (PRN) among 66 to 75 indexes was calculated for evaluation of normalisation performance (Hosomichi et al., 2014). The 
160 normalisation degree at $50 \%\left(\mathrm{ND}_{50}\right)$ was calculated as the ratio of single libraries of 161 which the PRNs were $50 \%$ or more of the average PRN.

162 


\section{Results}

164 Determination of a temperature range for MC assay

165 The short DNA or KAPA fragments were subjected to the MC assay. The results 166 indicated a large portion of the short fragments, of which the majority were less than $167300 \mathrm{bp}$ in length, dissociated into ssDNA at temperatures less than $86^{\circ} \mathrm{C}$, while the 452 $168 \mathrm{bp}$ fragment only began to dissociate at $86^{\circ} \mathrm{C}$ (Supplementary Figure S2a and S2b).

169 The short DNA and KAPA fragments were also subjected to the assay in two different 170 types of PCR buffers, demonstrating that components of the buffers slightly affected the 171 MT of both DNA fragments (Supplementary Figure S2c).

\section{Comparison of the library quantification methods}

174

175

176

177

Using parallel-processed sequencing libraries, quantification results of the MC assaybased method was compared with those of TapeStation-, and NanoDrop-based methods (Supplementary Figure S3). The sequencing libraries were prepared from 67 Epichloë festucae var. Iolii endophyte samples. Following adaptor ligation, the libraries were enriched through PCR. Monitoring of the amplification process on the real-time PCR instrument revealed that most samples reached the PCR plateau phase within 14 reaction cycles. Using another portion of the adaptor-ligated products, PCR amplification was performed for 12 cycles in the absence of fluorescence dye, to generate sequencing libraries with a range of dsDNA concentration. The concentration of the 12-cycled products was measured using each of the three methods: MC assay-, TapeStation-, and NanoDrop-based quantification (Fig. 1). The DNA library concentrations varied from 0 (undetectable) to $7.51 \mathrm{ng} / \mu \mathrm{l}$, obtained with the TapeStation-based method. The results from the three methods were correlated with 
187 one other $\left(R^{2}>0.77\right)$. Comparison of the three methods indicated that a total dRFU value

188 of 100 may be sufficient for relatively reliable quantification when the MC assay-based 189 method is used. The sum of dRFU values between 75 and $95^{\circ} \mathrm{C}$ exhibited a lower correlation with the other quantification results (Supplementary Figure S4).

191

192

193

194

195

196

197

198

199

200

201

202

\section{MC-based library quantification and normalisation}

The 14-cycled libraries were subjected to the MC assay (Fig. 2), revealing that the total dRFU values between 86 and $95^{\circ} \mathrm{C}$ varied less than those of the 12-cycled samples. $A$ value from a single sample was negative $(-5)$, indicating failure of library preparation. The sum of dRFU from the rest of samples was over 150. The 66 successful libraries were pooled for multiplexed sequencing, according to the quantification result (Supplementary Table 2). From the pooled library, 4.7 million paired-end reads were generated using a part of a sequencing run on the MiSeq platform. The PRNs of the 66 libraries varied from 0.45 to 3.21 , with an average of 1.52 , a standard deviation of 0.47 and coefficient of variation (CV) of 0.31 (Fig. 2). Except for the lowest library, the PRNs were over 0.77 , and the $\mathrm{ND}_{50}$ was consequently 0.98 . Based on the obtained sequencing data, PRNs in the absence of a normalisation procedure were also predicted. This simulation revealed that the PRNs from 8 libraries $(8 / 66=0.12)$ would be less than half of the average $(1.52 / 2=0.76)$, if an equal volume of libraries were pooled. The CV of PRNs for the un-normalised pool was calculated to be 0.38 .

For further validation, sequencing libraries were prepared from another set of 75 Epichloë festucae var. Iolii strains. Following library enrichment based on PCR, single libraries were subjected to the MC assay-based quantification procedure (Supplementary Figure S3). The total dRFU values from libraries varied from 20 to 3537 
211 (Fig. 3a), while that of the no-template PCR control was 15. From each library, the same

212 volume $(5 \mu \mathrm{l})$ of the PCR products was pooled and then purified with the AMPure XP 213 beads. The pooled library was loaded on the MiSeq platform, and a total of 1.4 million

214 reads were generated using a proportion of a MiSeq sequencing run. The PRNs of the 215 sequencing output were correlated with the total dRFU values $\left(R^{2}=0.82\right)$. A further 216 correlation analysis indicated that the total dRFU values for the temperature range 217 between 85 and $95^{\circ} \mathrm{C}$ were most strongly correlated to the PRNs $\left(R^{2}=0.823\right)$, followed 218 by the total dRFU values between 85 and $94^{\circ} \mathrm{C}\left(R^{2}=0.822\right)$, and between 86 and $95^{\circ} \mathrm{C}$ $219 \quad\left(R^{2}=0.821\right)($ Supplementary Figure S5).

A simulation study was also performed using these results. Assuming that the 221 total dRFU values between 86 and $95^{\circ} \mathrm{C}$ are in direct proportion to library 222 concentrations, volumes of the PCR products for pooling were calculated. As the total 223 dRFU values from two of the libraries were lower than 100 (20 and 26), these were 224 excluded from the simulation. The simulated PRNs were obtained based on the 225 calculated pooling volume and MiSeq output, and were between 0.5-2.27 (Fig. 3b). The 226 average PRN was 1.37 , and the $\mathrm{ND}_{50}$ and $\mathrm{CV}$ of PRNs were consequently 0.99 and 227 0.25 , respectively. No strong correlations were observed between total dRFU values 228 and PRNs after normalisation $\left(R^{2}=0.25\right)$. 


\section{Discussion}

230 PCR is commonly used as a target enrichment method for massively parallel

231 sequencing (Hosomichi et al., 2014, Hosomichi et al., 2015). The MspJl-based DNA

232 fragmentation method permits inexpensive sequencing library preparation from

233 amplicon-based templates (Shinozuka et al. 2015). A combination of use of these

234 techniques with the MiSeq platform allows low-cost sequencing analysis for the targeted

235 loci. For instance, DNA sequences with a cumulative length of $15 \mathrm{~kb}$ could be enriched

236 through PCR with locus-specific primers at a cost of US\$3.4/sample, and a single library

237 could be processed until the library enrichment stage for a cost of US\$3.3

238 (Supplementary Table S3). Libraries from up to 384 samples can be sequenced on the

239 MiSeq platform with the current Reagent Kit v3 (600 cycle; Illumina), generating an

240 average of approximately 2,000 times read depth for each nucleotide position within the

$24115 \mathrm{~kb}$-long regions. As the MiSeq kit costs less than US\$1,500, the per-sample cost for

242 this sequencing option would be under US\$4. Compared with these examples,

243 however, the per-sample costs of the existing high-throughput library normalisation

244 methods, are relatively high (Table 1) even though the primary purpose is solely DNA

245 quantification or normalisation. The MC assay-based library quantification procedure

246 costs substantially less than the other methods; only $1 / 3$ of the NanoDrop-based

247 method. The processing time of the MC assay-based method is also considerably

248 shorter than those of the other methods. For instance, 384 samples may be processed

249 within 3 hours on a standard real-time PCR instrument (4×96-well microtiter plates), and

250 the duration could be reduced to only 40 mins if a 384-well formatted instrument was

251 made available. The MC assay-based library quantification would, therefore, be most

252 suitable for sequencing experiments typified by large sample number but relatively 
253 small-output, such as HLA typing on the MiSeq platform (Hosomichi et al., 2015).

254 As the quantification results from MC assay-, TapeStation-, and NanoDrop255 based method were correlated with one other, each method can be used for the 256 purpose of library normalisation. Similar to the previously reported 'over-estimation 257 issue' (Simbolo et al. 2013), the quantification results obtained with the NanoDrop 258 system were, however, much larger than those obtained with the other methods, 259 especially when low-concentration libraries were subjected to analysis. Based on the 260 NanoDrop-based method, concentrations of $6.51-9.06 \mathrm{ng} / \mu \mathrm{l}$ were calculated from 4 261 library samples, from which no DNA was detected with the TapeStation-based method. 262 Similar results were observed when the result of NanoDrop-based quantification was 263 compared with that of the MC assay. As the quantification result from the blank sample 264 (10 mM Tris-HCl buffer without DNA) on the NanoDrop system was $0.17 \pm 0.25 \mathrm{ng} / \mu \mathrm{l}(3$ 265 replications), residual molecules from the SPRI-based DNA size-selection may have affected UV-visible spectrophotometry-based quantification.

During the MC assay, the target dsDNA and short DNA can be differentiated on 268 the basis of differences in MT (Ririe, Rasmussen \& Wittwer, 1997). The preliminary experiment with the short DNA and KAPA fragments suggested that the dRFU values at less than $86^{\circ} \mathrm{C}$ broadly represent the quantity of dsDNA shorter than $300 \mathrm{bp}$ in length, while those at and over $86^{\circ} \mathrm{C}$ represent the quantity of target DNA. In the subsequent experiments, sequencing libraries with the average fragment size of approximately 500 273 bp in length were subjected to the MC assay-based quantification, and the correlation between the total dRFU values for the temperature range 86 and $95^{\circ} \mathrm{C}$ and other quantification method was demonstrated. As the values between 75 and $95^{\circ} \mathrm{C}$ exhibited a lower level of correlation, exclusion of the fluorescence signals from short dsDNA is 
277 essential for accurate library quantification (Supplementary Figures S4 and S5). The

278 average fragment size of the sequencing library is determined in practice by multiple

279 factors, such as types of the sequencing platforms, sequencing reagents, library 280 preparation methods and experiment purposes. Optimisation of the temperature range 281 of the MC assay, therefore, may be required, depending on the average fragment size 282 of the sequencing library.

283 The length of DNA fragments is not the only factor determining the MT point, as

284 the GC-content ratio and sequence complexity are also relevant (Ririe, Rasmussen \&

Wittwer, 1997). Differences between sequencing libraries for these parameters may

286

287

288

289

290

291

292

293

294

295

296

297

298

299

300

hence influence accuracy of the MC assay-based quantification, and the method may not be suitable for a comparison of DNA quantities, if the DNA sequence contents are considerably different between the libraries. In general, libraries for multiplexed sequencing are, however, prepared in parallel from the identical targeted sequence(s) (Campbell, Harmon \& Narum, 2015; Hosomichi et al., 2014, Shinozuka et al. 2015), and the DNA sequence contents of libraries should not be substantially different. For the scenario of different DNA sequence contents, NanoDrop- or TapeStation-based methods would be more suitable.

The $\mathrm{CV}$ of $\mathrm{PRNs}$ and $\mathrm{ND}_{50}$ from the first normalisation experiment were 0.31 and 0.98 , respectively, and those from the second simulation experiment were 0.25 and 0.99 , respectively. The result of the MC assay-based method may hence be almost equivalent to that of the BeNUS (Bead-based Normalisation for Uniform Sequencing depth) method (CV of PRNs $\left.=0.31, N_{50}=0.93\right)$ (Hosomichi et al., 2014), although the normalisation result obtained with the qMiSeq method $(C V$ of $P R N s=0.05)$ was superior to the current result (Katsuoka et al., 2014). 
In this study, relatively accurate quantification results with the MC assay-based

302 method have been shown. Although the SYBR Green I fluorescent dye has been commonly used for a real-time PCR and MC assay in molecular biology laboratories, 304 the Eva Green (Biotium, CA, USA) and SYTO fluorescence dyes (Thermo Fisher Scientific) may provide better performance than SYBR Green I (Eischeid, 2011). Use of such fluorescence dyes may improve the library quantification performance of the MCbased method.

The BeNUS method permits high-throughput automated library normalisation, through reduction of the amount of SPRI beads necessary to capture a limited amount of DNA (Hosomichi et al., 2014). This method may consequently be ineffective when

311 library concentrations are too low. The results of the current study suggest that the MC 312 assay-based method performs better for recovery of libraries with low concentration. 313 Due to the high-throughput nature of both the BeNUS and MC assays, a combination of 314 the two may provide a high-quality automated normalisation procedure suitable for an 315 even larger sample volume, numbering in the several thousands. 


\section{Acknowledgments}

317 The authors would like to thank Dr. Emma Ludlow for provision of the perennial

318 ryegrass-associated fungal endophyte materials, and Dr. Noel Cogan for technical 319 advice.

320

321 Competing Interests Statement

The authors declare no competing interests 


\section{References}

323 Buehler B, Hogrefe HH, Scott G, Ravi H, Pabón-Peña C, O’Brien S, Formosa R, Happe S. 2010.

324 Rapid quantification of DNA libraries for next-generation sequencing. Methods 50:S15-S18. DOI:

325 10.1016/j.ymeth.2010.01.004.

326

327 Campbell NR, Harmon SA, Narum SR. 2015. Genotyping-in-Thousands by sequencing (GT-seq): A

cost effective SNP genotyping method based on custom amplicon sequencing. Molecular Ecology

Resources 15:855-867. DOI: 10.1111/1755-0998.12357.

330

331

332

Research Notes 4:263. DOI: 10.1186/1756-0500-4-263.

333

334

Harris JK, Sahl JW, Castoe TA, Wagner BD, Pollock DD, Spear JR. 2010. Comparison of Normalization Methods for Construction of Large, Multiplex Amplicon Pools for Next-Generation

336 Sequencing. Applied and Environmental Microbiology 76:3863-3868. DOI:

10.1128/AEM.02585-09.

Hosomichi K, Mitsunaga S, Nagasaki H, Inoue I. 2014. A Bead-based Normalization for 340 Uniform Sequencing depth (BeNUS) protocol for multi-samples sequencing exemplified by HLA-B. BMC Genomics 15:645. DOI: 10.1186/1471-2164-15-645.

342

Hosomichi K, Shiina T, Tajima A, Inoue I. 2015. The impact of next-generation sequencing 344 technologies on HLA research. Journal of Human Genetics. DOI: 10.1038/jhg.2015.102. 
346 Katsuoka F, Yokozawa J, Tsuda K, Ito S, Pan X, Nagasaki M, Yasuda J, Yamamoto M. 2014. An

347 efficient quantitation method of next-generation sequencing libraries by using MiSeq sequencer.

348 Analytical Biochemistry 466:27-29. DOI: 10.1016/j.ab.2014.08.015.

349

350

351

352

353

5141EN.pdf (accessed 11 April 2016)

354

355

356

K, Powers TL, Rizzolo K, Robbins D, Ryan E, Russ C, Sparrow T, Stalker J, Steelman S, Weiand M,

Zimmer A, Henn MR, Nusbaum C, Nicol R. 2010. A scalable, fully automated process for

construction of sequence-ready barcoded libraries for 454. Genome Biology 11:R15. DOI:

10.1186/gb-2010-11-2-r15.

361

Ririe KM, Rasmussen RP, Wittwer CT. 1997. Product Differentiation by Analysis of DNA Melting

Curves during the Polymerase Chain Reaction. Analytical Biochemistry 245:154-160. DOI:

10.1006/abio.1996.9916.

365 target capture. Genome Research 22:939-946. DOI: 10.1101/gr.128124.111.

368

Schmieder R, Edwards R. 2011. Quality control and preprocessing of metagenomic datasets. 
370 Bioinformatics 27:863-864. DOI: 10.1093/bioinformatics/btr026.

371

372 Shinozuka H, Cogan NOI, Shinozuka M, Marshall A, Kay P, Lin Y-H, Spangenberg GC, Forster JW.

373 2015. A simple method for semi-random DNA amplicon fragmentation using the methylation-

374 dependent restriction enzyme MspJI. BMC Biotechnology 15. DOI: 10.1186/s12896-015-0139-7.

375

376 Simbolo M, Gottardi M, Corbo V, Fassan M, Mafficini A, Malpeli G, Lawlor RT, Scarpa A. 2013. DNA

377 Qualification Workflow for Next Generation Sequencing of Histopathological Samples. PLoS ONE

378 8:e62692. DOI: 10.1371/journal.pone.0062692. 


\section{Table legend}

380

\section{Table 1}

382 Cost and processing duration assumptions for 92 to 96 samples using the library 383 normalisation or quantification methods. SPRI denotes the SPRI bead-based DNA size384 selection and purification before the quantification procedure. Size-specificity indicates 385 that the method is able to exclude both unnecessary long (e.g. $>1 \mathrm{~kb}$ ) and short (e.g. $386<300$ bp) DNA (++), or merely short DNA (+) from quantification. Library-specificity 387 indicates that the method detects only dsDNA with the sequencing adaptors on both ends (+), or also dsDNA without the sequencing adaptor(s), from which clonal sequence

389 clusters cannot be generated (-). Expenditure on instruments is not included in the per390 sample cost. More details can be found in Supplementary Table S1. 


\section{Figure Legends}

392

393

394

395

396

397

398

399

400

401

402

403 404

405

406

407

408

Fig. 1

Correlation analysis between the three library quantification methods: (a) MC assayand TapeStation-based methods; (b) MC assay- and NanoDrop-based methods; and (c) TapeStation- and NanoDrop-based methods.

\section{Fig. 2}

Results of the MC assay (a) and high-throughput sequencing (b) from the 66 successful libraries. The horizontal axis shows the sample unique identifier. In the result from highthroughput sequencing, the blue circle indicates PRN from the MiSeq-derived output, and the red square shows the simulated PRN for un-normalised multiplexed sequencing.

\section{Fig. 3}

Correlation between the MC assay-based quantification and PRN of MiSeq sequencing from the 75 samples (a), and between the MC assay-based method and predicted PRN from the library normalisation simulation from the 73 successful samples (b). 
409 Supplementary Data Legends

410

411 Supplementary Figure S1

412 Sequence and structure of in-house Y-shaped adaptor. (a) The PE1_adp_(+) and 413 PE2_adp_(-) oligonucleotides were ordered from Integrated DNA Technologies (IDT; IA, 414 USA). Asterisk $\left({ }^{*}\right)$ between nucleotides denotes a phosphorothioate modification. (b) 415 PE1_adp_(+) and PE2_adp_(-) oligonucleotides were annealed through incubation at $41694^{\circ} \mathrm{C}$ for 1 minutes, and subsequent cool to $37^{\circ} \mathrm{C}$ for 30 mins. Annealed nucleotides are 417 connected with a light blue line.

\section{Supplementary Figure S2}

420

421

422

Results of the TapeStation and MC assay. (a) The TapeStation results for the short DNA fragments, KAPA fragment and a single sequencing library, showing size distribution of dsDNA. The High Sensitivity D1000 kit (Agilent) was used for the short DNA and KAPA fragments. The $y=0$ and $x=0$ axes show the fluorescence intensity and DNA fragment size, respectively. 'Lower' and 'Upper' indicate signal peaks of upper and lower DNA size markers, respectively. (b) The melting peak plots from the three samples obtained for temperatures between 75 and $95^{\circ} \mathrm{C}$. (c) Effect of PCR buffer on MT of the short DNA and KAPA fragments. The MC assay was performed using three PCR buffers; the Phusion DNA polymerase HF and GC buffers and MyFiTM DNA polymerase buffer (Bioline, London, UK).

\section{Supplementary Figure S3}

Schematic explanation for the library quantification experiments and library pooling 
433 procedure.

434

\section{Supplementary Figure \$4}

436 Correlation analysis between the sum of dRFU values between 75 and $95^{\circ} \mathrm{C}$ and

437 quantification results from the TapeStation (a) and NanoDrop-based (b) methods.

\section{Supplementary Figure S5}

440 Coefficient of determination $\left(R^{2}\right)$ between the PRN from MiSeq sequencing and sum of dRFU values, depending on temperature ranges of the $M C$ assay. The vertical and horizontal axes denote lower and upper range of the temperatures $\left({ }^{\circ} \mathrm{C}\right)$ of the $\mathrm{MC}$ assay, respectively. The highest coefficient of determination $\left(R^{2}=0.823\right)$, which is from the PRN and dRFU values between 85 and $96^{\circ} \mathrm{C}$, is marked with an asterisk. websites of suppliers. Micropipette tips and 96-well microtiter plates are included in the consumables, and the per-sample cost for each of those was estimated as US\$0.04. SPRI denotes the SPRI bead-based DNA size-selection and/or purification.

\section{Supplementary Table $\mathbf{S 2}$}

454

Result of MC assay-based quantification and library normalisation procedure. A report file of the MC assay was exported from the CFX ConnectTM Real-Time PCR Detection System, and summation of dRFU between 86 and $95^{\circ} \mathrm{C}(\mathrm{A})$ was calculated for each 
457 single library. The sample ID 34 was excluded from the following procedure, as the 458 summation was below 100. The lowest summation was 157.6 (sample ID 53) among 459 the rest of samples. The summation of dRFU (A) was, then, divided with 157.6 (lowest 460 summation) to calculate relative concentration (B). The single libraries $(5 \mu \mathrm{l})$ were 461 diluted with $45 \mu \mathrm{l}$ water (total volume: $50 \mu \mathrm{l}$ ), and $45(\mu \mathrm{l})$ was divided with the relative 462 concentration (B) to obtain volume $(\mu \mathrm{l})$ to pool, which permitted pooling $45 \mu \mathrm{l}$ from the 463 lowest concentration sample. The single libraries were pooled following this result, and 464 pooled library was cleaned and concentrated with the AMPure XP beads. NTC and NA 465 stand for no-template control and not applicable, respectively.

\section{Supplementary Table S3}

468 Details for cost-reduced PCR amplicon sequencing library preparation with 469 fragmentation produced by the MspJl restriction endonuclease. A cumulative length of $47015 \mathrm{~kb}$ of DNA sequence can be enriched through 5 PCRs ( $3 \mathrm{~kb} \times 5$ reactions) using the 471 MyFiTM DNA polymerase. 
Table 1

474

\begin{tabular}{|c|c|c|c|c|c|c|}
\hline Method & Method type & $\begin{array}{c}\text { Size } \\
\text { specificity }\end{array}$ & $\begin{array}{l}\text { Library } \\
\text { specificity }\end{array}$ & $\begin{array}{l}\text { Per-sample } \\
\text { cost (US\$) }\end{array}$ & $\begin{array}{l}\text { Processing time for } \\
\text { 92-96 samples }\end{array}$ & Reference \\
\hline SequalPrep & Normalisation & $++^{*}$ & - & 0.91 & 1h30min & Campbell et al. (2015), Harris et al (2010) \\
\hline BeNUS & Normalisation & ++ & - & 1.04 & $1 \mathrm{~h} 30 \mathrm{~min}$ & Hosomichi et al. (2014) \\
\hline qMiSeq & Quantification & ++ & + & $\sim 10$ & c. 1 day & Katsuoka et al. (2014) \\
\hline $\begin{array}{c}\text { TapeStation } \\
\text { SPRI + real-time }\end{array}$ & Quantification & ++ & - & 2.75 & $2 \mathrm{~h} 15 \mathrm{~min}$ & Kong et al. (2014) \\
\hline PCR & Quantification & + & + & 1.41 & $4 \mathrm{~h} 50 \mathrm{~min}$ & Buehler et al. (2010), Rohland \& Reich (2012) \\
\hline SPRI + NanoDrop & Quantification & + & - & 0.48 & 1h50min & Harris et al. (2010) \\
\hline MC assay & Quantification & + & - & 0.15 & $40 \mathrm{~min}$ & \\
\hline
\end{tabular}




\section{$478 \quad$ Figure 1}

479

480 
a

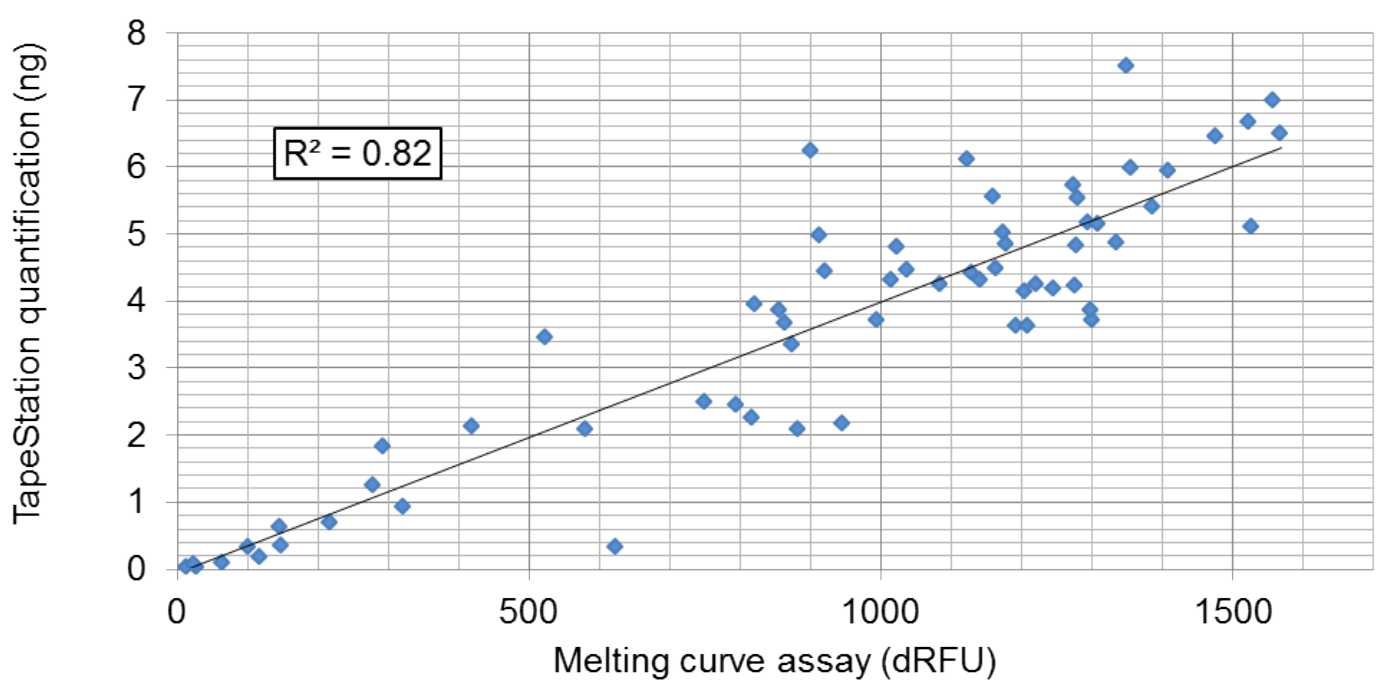

b
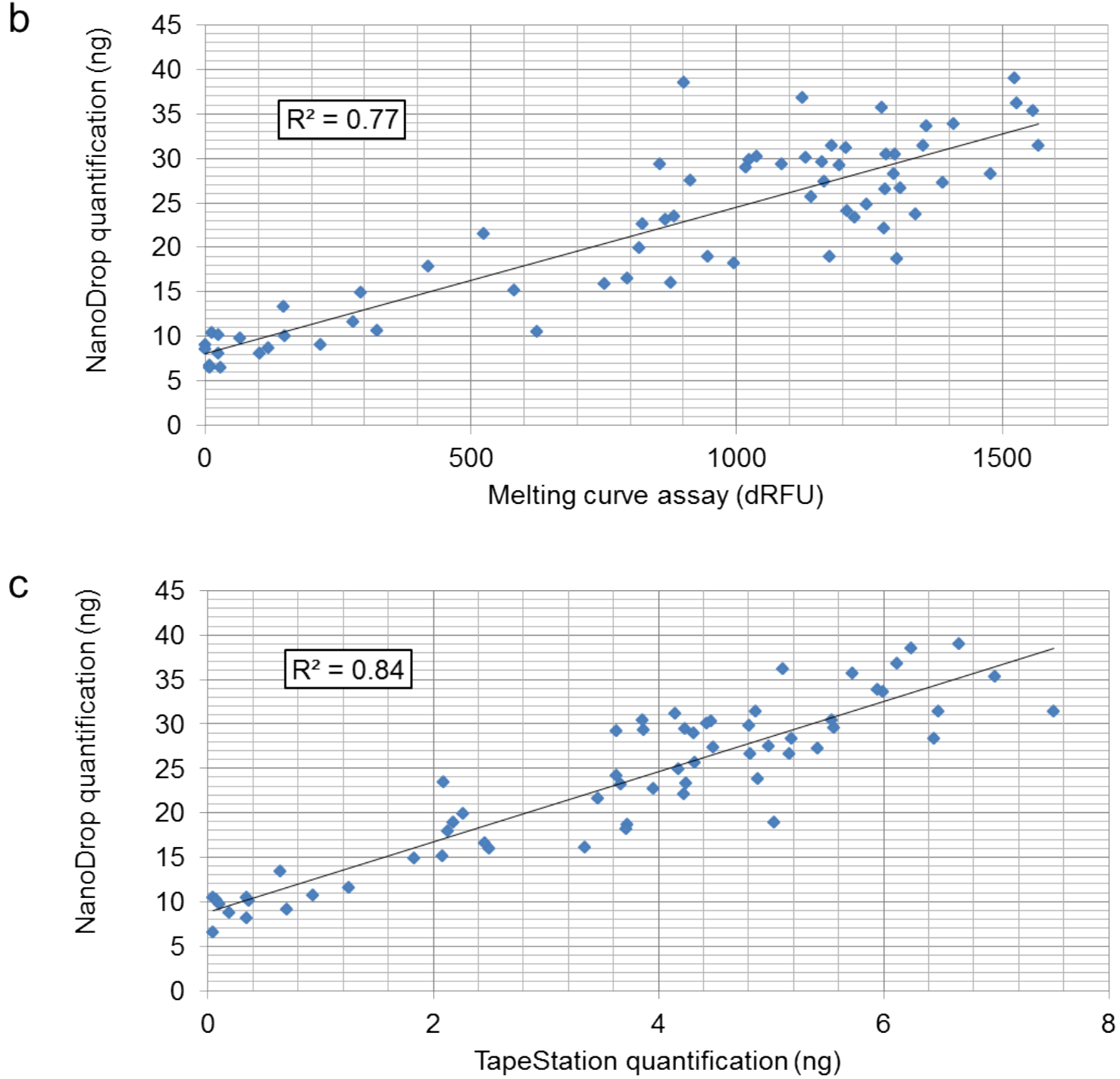


\section{Figure 2}

a

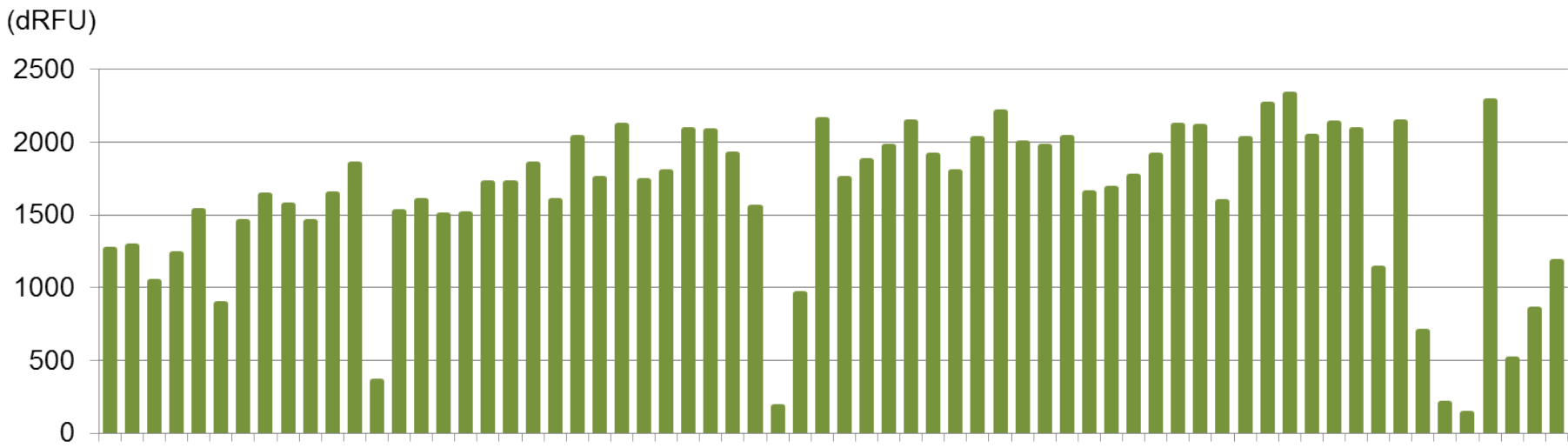

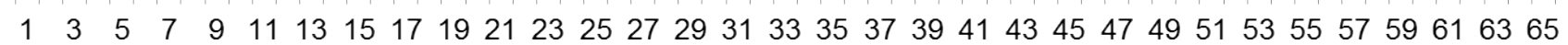

b (PRN)

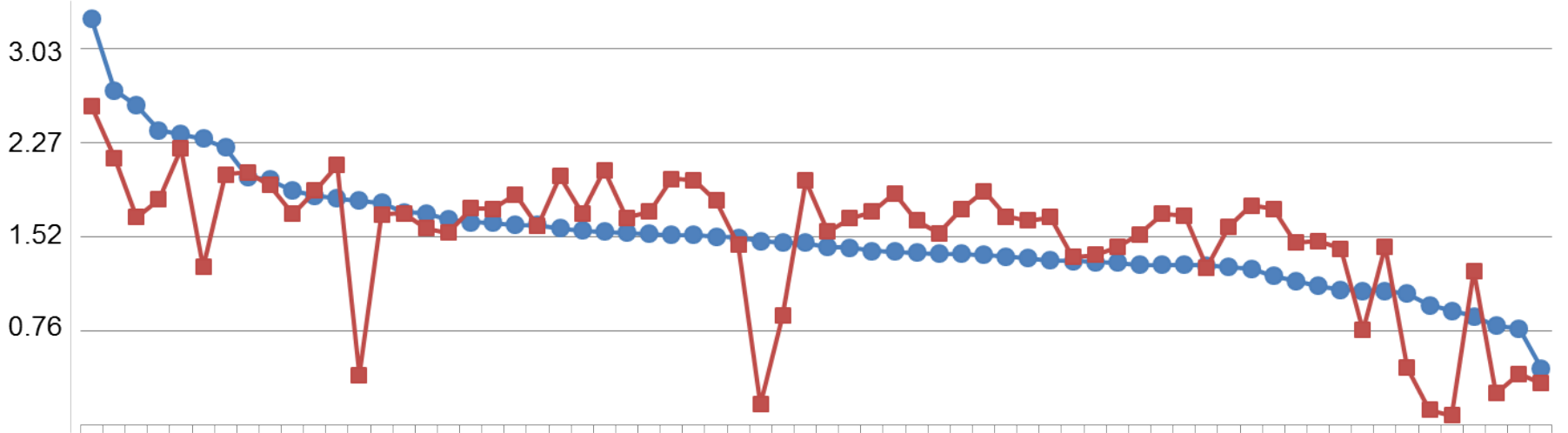

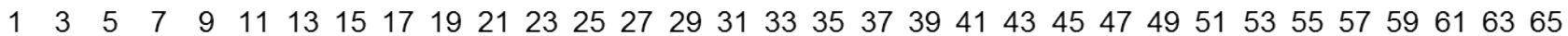




\section{Figure 3}

a

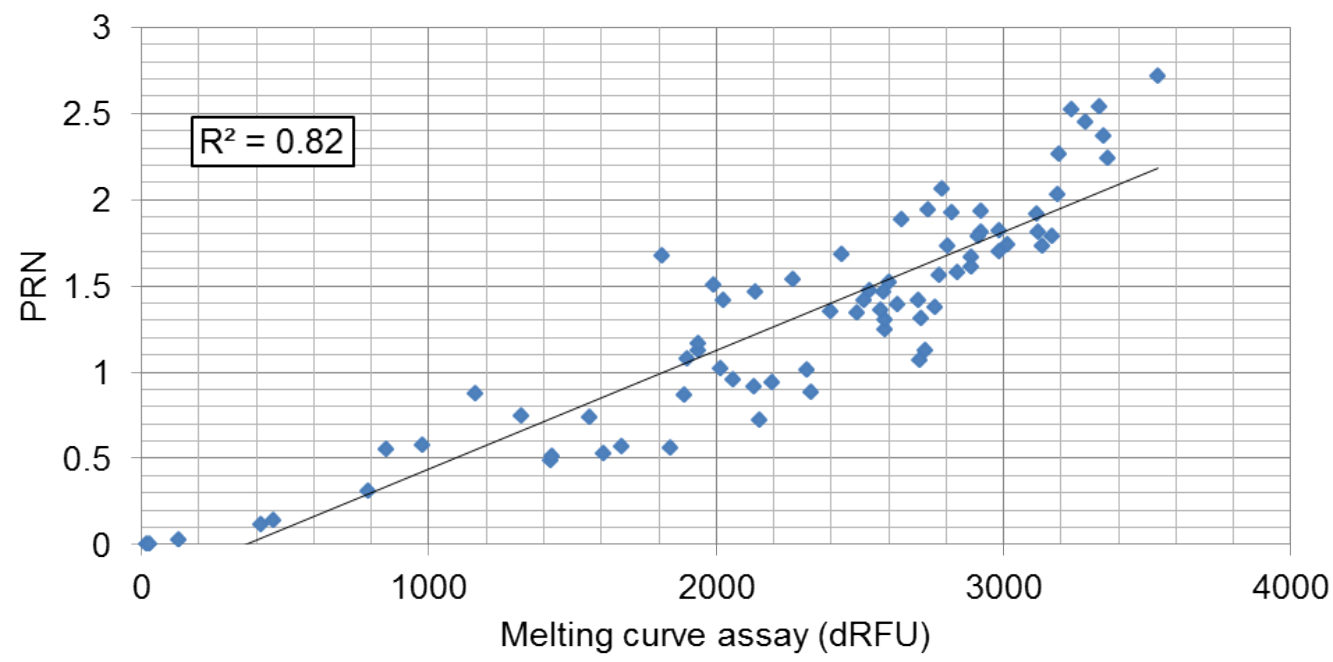

b

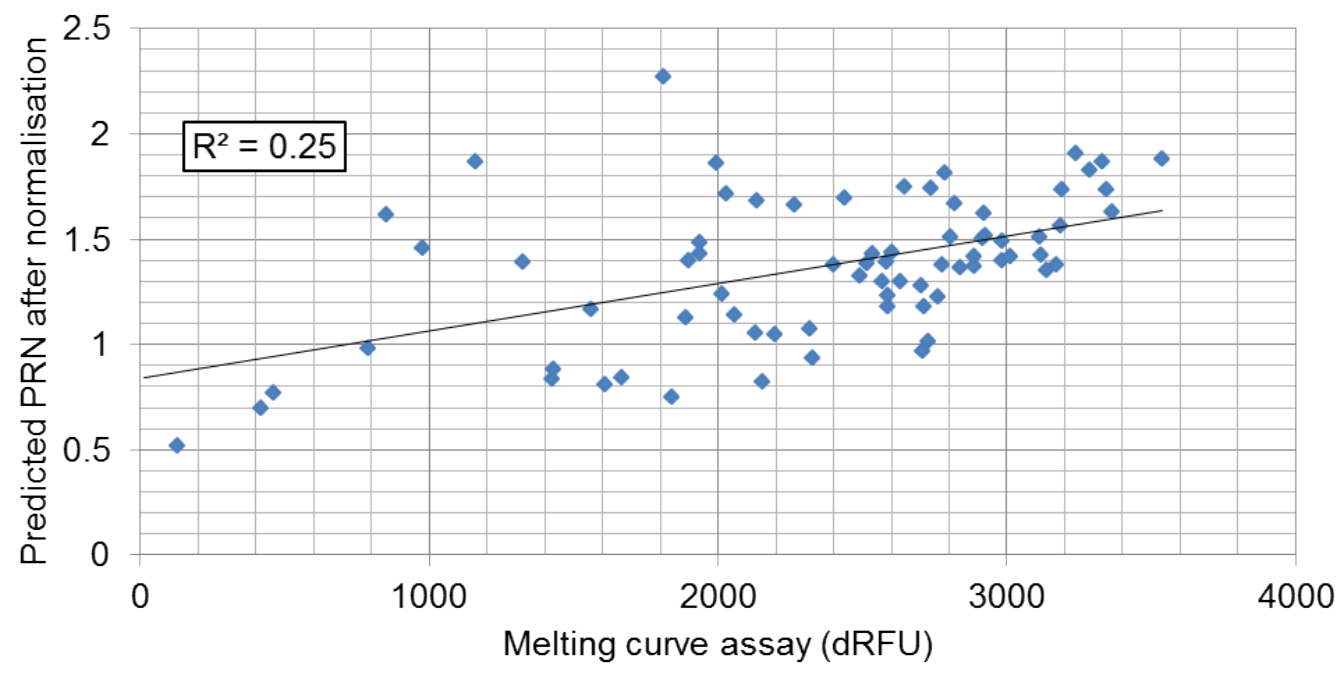

